\title{
ANÁLISE DA ORIGEM DAS AÇÕES JUDICIAIS CONTRA O ESTADO DE PERNAMBUCO PARA FORNECIMENTO DE MEDICAMENTOS ENTRE 2014 E 2016
}

\author{
C. C. de SOUZA ${ }^{1}$, T. de S. FIGUEIREDO² e D. M. GUEDES $^{3}$ \\ 1,2 Núcleo de Ações Judiciais da Secretaria Estadual de Saúde de Pernambuco \\ 3 Universidade Federal de Pernambuco, Programa de Pós-Graduação em Inovação \\ Terapêutica \\ E-mail para contato: camila4932@gmail.com
}

\begin{abstract}
RESUMO - O direito ao acesso a medicamentos essenciais está consolidado no Brasil, no entanto há problemas relacionados à gestão. A judicialização da saúde é uma realidade que precisa ser analisada pelos gestores em saúde. Este estudo busca identificar unidades de saúde que dão origem às ações judiciais para fornecer medicamentos. Foi realizado levantamento das ações judiciais contra o Estado de Pernambuco entre 2014 e 2016. Foram identificadas as unidades de saúde que demandaram ações judiciais, classificadas em filantrópicas, unidades do SUS e unidades particulares. Verificou-se que 31,1\% das ações vêm de entidades filantrópicas, 36,7\% são do SUS e 27,4\% são de unidades privadas, portanto a maioria das ações judiciais é originada em unidades do SUS. Os resultados coincidem com outros estudos realizados anteriormente.
\end{abstract}

PALAVRAS CHAVE: Judicialização da Saúde. Unidades de Saúde. Acesso a medicamentos. Direito à Saúde.

\begin{abstract}
The right to access essential medicines is consolidated in Brazil, but there are problems related to management. The judicialization of health is a reality that needs to be analyzed by health managers. This study seeks to identify health units that give rise to lawsuits to provide medicines. A survey of lawsuits against the State of Pernambuco was carried out between 2014 and 2016. Health units were identified that demanded lawsuits classified as philanthropic, public and private units. It was verified that $31.1 \%$ of the actions come from philanthropic entities, $36.7 \%$ are from Public units and $27.4 \%$ from private units, so most of the lawsuits are originated in Public units. The results agree with previous studies.
\end{abstract}

KEYWORDS: Health Judicialization. Health Units. Access to medicines. Right to Health.

\section{INTRODUÇÃO}

No Brasil, a Lei $n^{0}$ 8.080/90 determina que a formulação da política de medicamentos passa a fazer parte do âmbito de atuação do Sistema Único de Saúde (SUS) e, a partir deste 
marco legal, a assistência farmacêutica foi sendo estruturada e hoje conta com importantes ferramentas como a Relação Nacional de Medicamentos (RENAME) e a Comissão Nacional de Incorporação de Tecnologias no SUS (CONITEC). Neste contexto, pode-se considerar superado o argumento que as listas de medicamentos do SUS não são revistas, já que a atualização dessas políticas conta com a participação da sociedade e são construídas a partir de conhecimentos técnico-científicos disponíveis (BARROSO, 2009).

A política pública de fornecimento de medicamentos representa importante instrumento de acesso equânime da população a esses produtos, garantindo o atendimento do direito humano e fundamental à saúde. No entanto, existem as omissões, especialmente nos medicamentos utilizados em doenças raras que ainda não possuem Protocolo Clínico e Diretrizes Terapêuticas; ainda, existem problemas na efetivação das políticas públicas causados por problemas na gestão, como falta de medicamento, atraso na entrega, etc. (BARROS, 2016).

O crescente número de ações judiciais propostas por usuários do SUS para garantir o direito à assistência farmacêutica tem sido motivo de preocupação para os gestores de saúde, e estes números estão causando inegável impacto nas contas públicas e dificultado a consecução dos princípios organizacionais do SUS. No entanto, a judicialização em si não é boa nem ruim; no contexto de universalidade e integralidade do acesso, ela deve ser compreendida como um fenômeno e a partir dessa análise, deve-se repensar as falhas de gestão do SUS.

Esta pesquisa tem como objetivo identificar as unidades de saúde que dão origem às ações judiciais contra o Estado de Pernambuco, cujo objeto seja o fornecimento de medicamentos, entre os anos de 2014 e 2016. A partir do mapeamento das unidades de saúde e ao classificá-las pela personalidade jurídica das mesmas, pode-se juntamente com outros estudos buscar subsídios para a gestão estadual para o enfrentamento da judicialização da saúde.

\section{MATERIAIS E MÉTODOS}

Foi realizado um levantamento das demandas judiciais cujo réu foi o Estado de Pernambuco entre os anos de 2014 e 2016, e que o objeto da ação judicial seja o fornecimento de medicamentos. Os dados obtidos a partir da leitura dos autos dos processos e seus anexos foram tabulados em planilha eletrônica. Os dados extraídos foram informações pessoais dos pacientes, patologia, nome do medicamento, profissional de saúde prescritor e unidade de saúde de origem.

A partir das informações obtidas, foram identificadas as unidades de saúde que demandaram ações judiciais contra o Estado de Pernambuco a fim de fornecer medicamentos. Estas unidades foram classificadas em filantrópicas, unidades do SUS e unidades particulares. Ainda, foram contabilizadas as demandas que não tiveram sua origem identificada, ou porque ele não existia nos autos ou porque estava ilegível. Os dados obtidos foram separados pelo ano em que foi dada a decisão liminar ou sentença judicial.

\section{RESULTADOS E DISCUSSÃO}

Ao realizar a análise dos dados obtidos, observou-se que foram 1310 pedidos de 
medicamentos em 2014, 1476 pedidos em 2015 e 1539 pedidos em 2016. No total acumulado, foram feitos 4325 pedidos de medicamentos nestes três anos. No entanto, é importante ressaltar que em uma única decisão judicial, pode haver em seu objeto a obrigação de fornecer vários medicamentos e outros produtos de saúde para o mesmo paciente. Assim, para viabilizar o dimensionamento das unidades de saúde de origem do paciente, foi considerado o número de ações judiciais cujo objeto contivesse no mínimo um medicamento. O número total de ações judiciais sob estes critérios e a quantidade de medicamentos pleiteados estão elucidados na Tabela 1.

Tabela 1 - Número de ações judiciais com medicamento como objeto, nos anos de 2014 a 2016.

\begin{tabular}{ccc}
\hline Ano & $\mathbf{N}^{\mathbf{0}}$ de ações judiciais & Quantidade de medicamentos \\
\hline $\mathbf{2 0 1 4}$ & 1073 & 1310 \\
$\mathbf{2 0 1 5}$ & 1172 & 1476 \\
$\mathbf{2 0 1 6}$ & 1130 & 1539 \\
\hline Total & $\mathbf{3 3 7 5}$ & $\mathbf{4 3 2 5}$ \\
\hline
\end{tabular}

No universo das unidades de saúde que originaram as demandas judiciais contra o Estado de Pernambuco, foi possível categorizar pela personalidade jurídica da entidade, classificando-as em filantrópicas, unidades públicas (SUS) e unidades privadas. Assim, cada ação judicial foi classificada como oriunda de uma dessas três categorias de unidade de saúde (SUS, privada ou filantrópica). Ainda, foram consideradas também as ações judiciais nas quais não foi possível determinar a unidade de saúde demandante, classificadas como "não informado". Com isso, pode-se determinar a distribuição percentual por cada categoria de unidade de saúde, conforme indicado na Figura 1.

Figura 1 - Percentual das unidades de saúde que demandaram medicamentos (2014 a 2016).

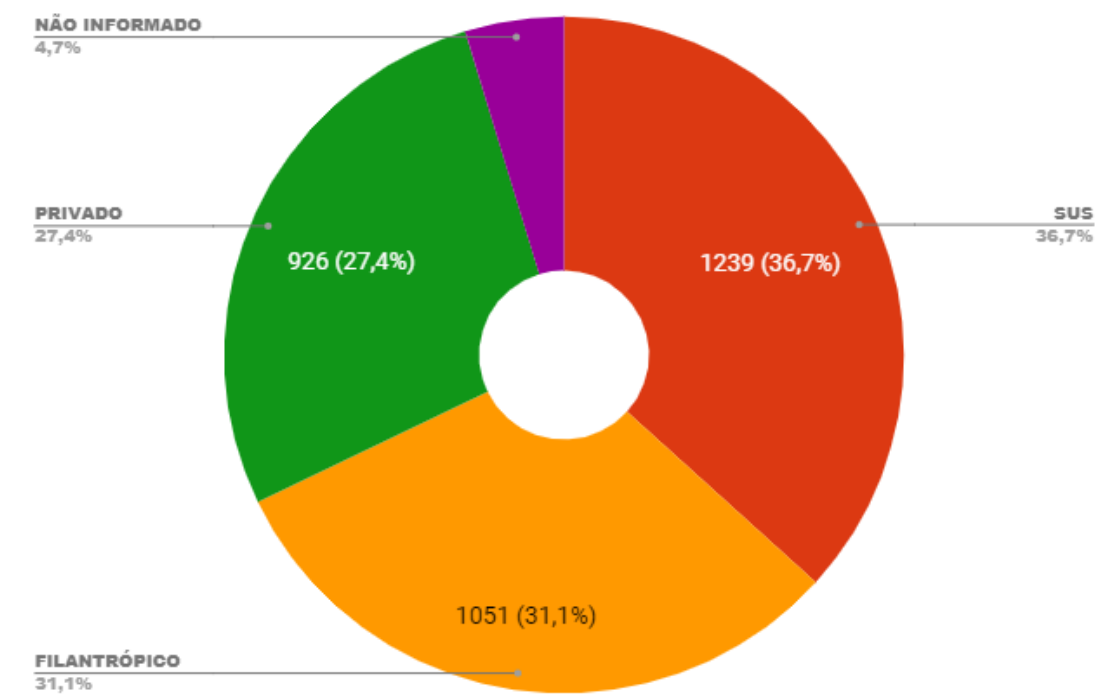

Considerando que os hospitais filantrópicos são em sua grande maioria vinculados ao SUS por convênio, temos em torno de $68 \%$ das demandas oriundas do SUS. Estes dados encontrados coincidem com as informações relatadas por Tabosa (2010), que verificou que a prescrição da demanda judicial à assistência farmacêutica do Estado de Pernambuco tem 77\% de origem no serviço público de saúde (55\% prescrito por médico de hospital público e $22 \%$ 
por médico do SUS) entre 2004 e primeiro semestre de 2009.

Ainda, os estudos de Nunes e Silva Júnior (2016) indicaram que as prescrições médicas que fundamentavam as decisões judiciais, na sua maioria, vinham da própria rede pública (76\%), enquanto $21,1 \%$ provinham da rede privada de saúde. Em 25 processos $(2,6 \%)$ não foi possível identificar a origem. Este trabalho avaliou as demandas judiciais do Estado do Ceará entre 1998 e 2012. Ainda segundo os autores, a revisão da literatura e os processos analisados mostraram que as prescrições médicas vêm preferencialmente do sistema público de saúde, embora existam também estudos em que há maior frequência das prescrições privadas.

\section{CONCLUSÃO}

Com este trabalho, foi possível identificar as unidades de saúde demandantes de medicamentos através ações judiciais no Estado de Pernambuco. Este mapeamento permitiu concluir que a maioria das ações judiciais contra o Estado são originadas em unidades de saúde vinculadas ao SUS, o que pode indicar, juntamente com estudos adjuntos, os gargalos no acesso a medicamentos que já constam na RENAME e também para apontar possíveis atrasos na incorporação de novas tecnologias no SUS, que acabam impactando no número de ações judiciais demandadas.

Os resultados demonstram a necessidade de mapear os fatores de crescimento da demanda por medicamento através do Poder Judiciário, assim como a execução da Política Nacional de Medicamentos, com o fim a auxiliar nas decisões do Executivo para o afastamento ou diminuição da despesa excepcional com assistência farmacêutica e suas implicações no orçamento para a saúde e, ao mesmo tempo garantir o acesso do cidadão à assistência farmacêutica de forma igualitária.

\section{REFERÊNCIAS}

BARROS, L. D. Judicialização do Direito à Saúde: uma análise acerca do fornecimento judicial de medicamentos no Estado de Pernambuco. Dissertação de Mestrado do Programa de Pós-graduação em Direitos Humanos da Universidade Federal de Pernambuco. Recife, 2016.

BARROSO, L. R. O direito constitucional e a efetividade de suas normas: limites e possibilidades da Constituição brasileira. 9. ed. Rio de Janeiro: Renovar, 2009.

NUNES, C. F. O.; RAMOS JÚNIOR, A. N. Judicialização do direito à saúde na região Nordeste, Brasil: dimensões e desafios. Cad. Saúde Colet., v. 24 (2). p. 192-199, 2016.

TABOSA, T. M. S. A Judicialização da Saúde no Estado de Pernambuco: Análise do Impacto das Decisões Judiciais sobre o Orçamento Público. Dissertação de Mestrado Profissional do Programa de Pós-graduação em Economia da Universidade Federal de Pernambuco. Recife, 2010.

\section{AGRADECIMENTOS}

Agradecemos ao Superintendente de Ações Judiciais da Secretaria Estadual de Saúde de Pernambuco, pelo apoio e orientações para o desenvolvimento da presente pesquisa. 